\title{
Detection of Subclinical Left Ventricular Systolic dysfunction by 2D Speckle Tracking Echocardiography in Patients with Peripheral Artery Disease
}

\author{
Mohammad S. Al-Baz ${ }^{1}$, Wael Mohammad Attia ${ }^{1}$, Hany A. Abd El-Fattah ${ }^{2}$, Islam F. Abd Al- \\ Gawad $^{1} *$ \\ ${ }^{1}$ Department of Cardiovascular Medicine, ${ }^{2}$ Vascular Surgery, Faculty of Medicine, Al-Azhar \\ University, Cairo, Egypt
}

*Corresponding author: Islam F. Abd Al-Gawad; Mobile: 01024330323; Email: dr.abonasab@gmail.com

\begin{abstract}
Background: peripheral arterial disease (PAD) is estimated to affect 202 million people worldwide, with prevalence that is increasing over time. PAD represents a local manifestation of systemic atherosclerosis and portends a 2-6 fold increase in both cardiovascular and cerebrovascular events with an annual mortality rate of $4-6 \%{ }^{(1,2)}$.
\end{abstract}

Aim of the Work: the aim of this work was to detect left ventricular subclinical dysfunction using 2dimensional speckle-tracking echocardiography (2D-STE) in patients with peripheral arterial disease.

Patients and Methods: the ethical approval was obtained from the hospital Ethical Research Committee and each patient entering the study signed an informed consent. In a case-control study, 30 patients included were diagnosed with peripheral arterial disease and 20 control. They were recruited from Vascular department at Al-Hussein and Bab El-She'riya University Hospitals during the period from June 2018 to November 2018.

Results: there was moderately strong significant correlation between $\mathrm{ABI}$ and left ventricle $2 \mathrm{~d}$ speckle tracking echocardiography (2D-STE) (GLS\% ( $\mathrm{p}=0.003, \mathrm{r}=0.52), \mathrm{AP} 4 \%(\mathrm{p}=0.001, \mathrm{r}=0.57)$, AP3\% ( $\mathrm{p}=$ $0.004, \mathrm{r}=0.51)$ and AP2\% ( $\mathrm{p}=0.005, \mathrm{r}=0.50)$ ). This correlation was obvious according to limb affection (one limb vs. both limbs, GLS\% p $=0.025$, AP4\% p $=0.038$, AP3\% $=0.013$ and AP2\% $=0.046$ ) and severity (severe arterial disease vs. moderate arterial disease vs. some arterial disease subgroups GLS\% $\mathrm{p}=0.003$, $\mathrm{AP} 4 \% \mathrm{p}=0.001, \mathrm{AP} 3 \%=0.002$ and $\mathrm{AP} 2 \%=0.015)$. Also, there was moderately strong significant correlation of ABI with left ventricle systolic function ( $\mathrm{p}=0.011, \mathrm{r}=0.46)$ while other conventional echo parameters were of no significance with decline in ABI.

Conclusion: peripheral arterial disease patients had lower global and segmental longitudinal strain than the healthy subjects and this was more prominent either with severity or limb affection. This study showed that even there is correlation between decline ABI and left ventricular systolic function EF\% but LV GLS offer a better economic way to detect subclinical dysfunction in asymptomatic patient.

Keywords: echocardiography, peripheral arterial disease, ankle brachial index and speckle tracking, global longitudinal strain.

\section{INTRODUCTION}

Peripheral arterial disease $(\mathrm{PAD})$ represents a local manifestation of systemic atherosclerosis $^{(2)}$ and portends a $2-6$ fold increase in both cardiovascular and cerebrovascular events. The diagnosis is also associated with an annual mortality rate of 4 $6 \%$. In addition to causing lifestyle limiting claudication symptoms, uncontrolled disease can progress on to critical limb ischemia the end stage of $\mathrm{PAD}^{(\mathbf{1})}$. The ankle-brachial index has been used as an important indicator for diagnosis of peripheral arterial disease, particularly in studies of elderly populations with a high incidence of clinically significant atherosclerotic disease. A low ABI is also associated with higher risk of cerebrovascular disease, more severe coronary artery disease, improved prediction of acute coronary events, higher prevalence of atherosclerosis ${ }^{(3)}$.

Approximately 202 million people are affected with PAD worldwide, of whom almost 40 million are living in Europe. Eight to ten million Americans suffer from PAD with an overall prevalence $12 \%$ in the adult population ${ }^{(4)}$. Regarding the natural history, in a recent meta-analysis, most patients with Intermittent Claudication present increased 5year cumulative CV-related morbidity of $13 \%$ vs. $5 \%$ in the reference population. Regarding the limb risk, at 5 years, $21 \%$ progress to Critical Limb Ischemia, of whom 4-27\% have amputations $^{(5)}$. Leg amputation due to 
atherosclerotic PAD gives rise to an acute mortality rate of approximately $30 \%$ and a 5year survival rate of less than $30 \%{ }^{(6,7)}$.

Although the reason for this poor prognosis for patients with PAD is unknown, it may be explained that a higher prevalence of co-existent coronary artery disease (CAD) in patients with $\mathrm{PAD}$ has frequently been mentioned as the primary cause for this high mortality rate thus decreased attention to additional or associated cardiac findings that may contribute to this poor prognosis despite prospective studies that showed and suggested that a low ABI predicts all-cause mortality even in subgroups of patients without established CAD. This make sense as coronary arteries and peripheral arteries have different embryological origin $^{(8-11)}$. But limited data are available on the association between echocardiographic findings and $\mathrm{PAD}^{(\mathbf{1 2 , 1 3 )} \text {. }}$

The coexistence of CAD and PAD was described since 1960. Peripheral arterial disease. PAD whether symptomatic or asymptomatic, is a risk factor for non-fatal and fatal coronary disease and cerebrovascular

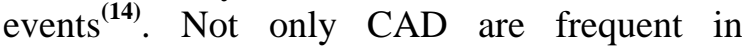
patients with PAD, but also this is true for heart failure, atrial fibrillation and left ventricular hypertrophy ${ }^{(2,15)}$.

The development of heart failure represents a late event in the cardiovascular variety and is largely the result of the concerted influence of risk factors that mediate atherosclerosis. Risk factors for HF (i.e., atherosclerosis, smoking, hypertension, and diabetes) cluster in patients with PAD. The presence of PAD is associated with a nearly 2fold increase in the prevalence of $\mathrm{HF}^{(16)}$. So, the new guidelines of ESC for PAD recommends not only, screening for heart failure in patients with symptomatic PAD should be considered but also screening for PAD may be considered in heart failure patients specially if is indicated for heart transplantation or a cardiac assist device, transcatheter aortic valve implantation or other structural interventions. Such coexistence may carry important prognostic and therapeutic implications and often needs a multidisciplinary approach ${ }^{(2)}$.

Echocardiography is the leading cardiac imaging technique in patients with suspected cardiac disease however; at rest it provides little information regarding the presence and extent of $\mathrm{CAD}^{(\mathbf{1 7 )}}$. Left ventricular (LV) function can be evaluated using directional components of myocardial deformation or strain. Longitudinal LV strain (also referred to as global longitudinal strain, GLS) appears to be a sensitive measure of impaired LV systolic function ${ }^{(\mathbf{1 8})}$ and has been shown in several studies to be better than ejection fraction at predicting cardiovascular disease events and death ${ }^{(\mathbf{1 9 - 2 1 )}}$.

\section{AIM OF THE WORK:}

The aim of this work is to detect left ventricular subclinical dysfunction using 2dimensional speckle-tracking echocardiography (2D-STE) in patient with peripheral arterial disease.

\section{PATIENTS AND METHODS}

The ethical approval was obtained from the hospital Ethical Research Committee and each patient entering the study signed an informed consent. In a case-control study, 30 patients diagnosed with peripheral arterial disease was included and 20 normal control. They were recruited from Vascular department at $\mathrm{Al}$ Hussein and Bab El-She'riya University Hospitals during the period from June 2018 to November 2018. In this study we assessed the patients by conventional echocardiography and 2D speckle tracking echocardiography of left ventricle.

\section{Case group (group A); case selection}

a) Inclusion criteria: Patients with documented peripheral arterial disease attending vascular surgery clinic diagnosed by $\mathrm{ABI} \leq 0.9$. $^{\text {(2) }}$

b) Exclusion criteria: Documented IHD, patient with history of cardiac intervention (PCI or CABG), patients with moderate or severe valvular disease, patients with conduction abnormalities, pacemaker, ongoing arrhythmia and bundle branch block, patients with congenital heart disease, patients with reduced $\mathrm{EF} \leq 50 \%$ or cardiomyopathy, atrial fibrillation, end stage renal disease, poor echocardiographic window, and patient refusal.

c) Classification: Patient group was classified according to limb affection into one limb affection and both limb affection. Also, it was classified according to ABI into some arterial disease (ABI $0.80-0.89$ ), moderate arterial disease (ABI $0.5-0.79)$ and severe arterial disease $(\mathrm{ABI}<0.5){ }^{(2)}$ 
Control group (group B): Twenty healthy individuals, age and sex matched, were used as a control group.

\section{Methods:}

(1) Clinical evaluation: included full history taking, general and local examination.

(2) Resting surface 12 ECG leads for analysis for any of exclusion criteria.

(3) Transthoracic echocardiogram (TEE): Based on recommendations on the echocardiographic assessment of chamber quantification guidelines ${ }^{(22)}$ :

a) Standard trans-thoracic echocardiographic study (TEE): TEE was performed using S4-2 transducer 4-2 $\mathrm{MHz}$ with a commercially available ultrasound system (Affiniti 50; Philips, Andover, MA, USA) according to the standardized protocol. LV internal dimensions, LV end-diastolic dimensions, and end-systolic dimensions, LV EF by Biplane Simpson's method.

b) 2D strain imaging by speckle tracking (2D-STE): 2D echocardiography images will be obtained from apical four, apical three and apical two chamber views. All images will be stored in cine-loop format from three consecutive beats. The frame rate for images was between 50 and 90 frames/s while analysis was performed off-line using commercially available software (Philips QLAB Advanced Quantification Software version 8.1).

(4) Ankle brachial index: The blood pressure cuff is inflated proximal to the artery in question and measured by the Doppler probe (5-8 MHZ), the inflation continues until the pulse in the artery ceases then, pressure cuff was slowly deflated. When the artery's pulse is re-detected through the Doppler probe the pressure in the cuff at that moment indicates the systolic pressure of that artery. The pressures in each foot's posterior tibial artery and dorsalis pedis artery are measured with the same way then, ABI was calculated ${ }^{(2)}$. $\mathrm{ABI}$ is defined as the ratio of the systolic blood pressures of the 2 ankle arteries of that limb (either the dorsalis pedis or the tibial artery) and the systolic blood pressures of the upper limb. We used $\mathrm{ABI}$ average of both limbs as some other authors preferred ${ }^{(7)}$.

\section{Statistical analysis:}

Data were analyzed using the computer program SPSS (Statistical package for social science) version 20. Categorical variables were expressed as frequency and parentage. Chi-square test was used when comparing between Categorical variables. Continuous variables are reported as mean \pm SD (standard deviation) and compared by $t$ test ( 2 groups comparisons) or ANOVA ( $>2$ groups comparisons). P-value $<0.05$ was considered significant, P-value $<0.001$ was considered highly significant, while $\mathrm{P}$-value $>0.05$ was considered insignificant.

\section{RESULTS:}

Among 50 participants in this study, 33 $(66 \%)$ were male and 17 (34\%) were female, 19 $(38 \%)$ were smokers, $14(28 \%)$ had diabetes mellitus, $11(22 \%)$ had hypertension, $3(6 \%)$ had family history mean age was $62.18 \pm 7.18$ years. While among 30 participants in case group in terms of PAD severity, 13 (43\%) had one limb affection, 17 (57\%) had both limb affection, 4 (13\%) had some arterial disease, 19 (63\%) had moderate arterial disease and 7 (23\%) had severe arterial disease.

Regarding to comparison between baseline characteristics between the case and the control groups, there was no statistically significant difference between patients and control as regard age $(\mathrm{P}$-value $=0.26)$, gender $(\mathrm{P}$-value $=0.229)$, diabetes mellitus (P-value $=0.118)$, hypertension $(\mathrm{P}$-value $=0.163)$, smoking $(\mathrm{P}$ value $=0.148$ ), and family history (P-value $=0.265$ ).

Also, regarding to characteristics of study parameters mean of ABI average was $0.74 \pm 0.23$, mean LV_EDD was $4.37 \pm 0.22$, mean LV_ESD was $3.22 \pm 0.17$, mean LV_EDV was $84.00 \pm 12.20$, mean LV_ESV was 33.78 \pm 5.32 , mean $\mathrm{EF} \%$ was $59.54 \% \pm 4.89 \%$, mean AP3\% was $-17.71 \pm-3.32$, mean AP4\% was $18.25 \pm-3.73$, mean AP2\% was $-17.94 \pm-3.30$ and mean GLS was -18.02 \pm-3.27 .

There was statistically significant difference between patients and control as regard ABI (P-value < 0.0001), 2D-STE parameter $(\mathrm{P}$-value $<0.0001)$ but there was no statistically significant difference between patients and control as regard other conventional echocardiography parameters (table 1). 
There was statistically significance $(\mathrm{P}-$ value $=0.011)$ between left ventricle systolic function $\mathrm{EF} \%$ and $\mathrm{ABI}$ average with a moderately strong negative correlation between both parameter $(\mathrm{r}=-0.46)$ (fig.2) and this significance was more affected in severe arterial disease than moderate arterial disease than some arterial disease subgroups (P-value= 0.043) (table1).

\section{Table 1: Shows study parameter in different groups}

\begin{tabular}{|c|c|c|c|c|c|c|c|}
\hline & & \multicolumn{3}{|c|}{ Study groups } & \multicolumn{3}{|c|}{ Limb affection subgroup } \\
\hline & & Patient & Control & \multirow{2}{*}{ P-Value } & One Limb & Both Limb & \multirow{2}{*}{ P-Value } \\
\hline & & Mean \pm SD & Mean \pm SD & & Mean \pm SD & Mean \pm SD & \\
\hline \multicolumn{2}{|l|}{ Age } & $62.57 \pm 7.53$ & $61.60 \pm 6.76$ & 0.646 & $60.54 \pm 8.84$ & $64.12 \pm 6.18$ & 0.202 \\
\hline \multirow{5}{*}{$\begin{array}{l}\text { Conventional } \\
\text { Echo. }\end{array}$} & $L V \_E D D$ & $4.34 \pm 0.26$ & $4.41 \pm 0.15$ & 0.238 & $4.33 \pm 0.27$ & $4.35 \pm 0.25$ & 0.846 \\
\hline & $L V_{-} E S D$ & $3.19 \pm 0.16$ & $3.27 \pm 0.18$ & 0.128 & $3.14 \pm 0.14$ & $3.23 \pm 0.16$ & 0.109 \\
\hline & $L V \_E D V$ & $82.44 \pm 13.92$ & $86.35 \pm 8.85$ & 0.271 & $81.85 \pm 14.46$ & $82.88 \pm 13.92$ & 0.845 \\
\hline & $L V \_E S V$ & $32.80 \pm 4.82$ & $35.25 \pm 5.80$ & 0.111 & $31.15 \pm 1.16$ & $34.06 \pm 5.02$ & 0.102 \\
\hline & $E F \%$ & $59.70 \pm 5.13$ & $59.30 \pm 4.62$ & 0.78 & $61.23 \pm 5.78$ & $58.53 \pm 4.40$ & 0.157 \\
\hline \multirow{4}{*}{$\begin{array}{l}\text { 2D Speckle } \\
\text { Echo. }\end{array}$} & AP3\% & $-15.54 \pm-2.32$ & $-20.98 \pm-1.22$ & $<0.0001$ & $-16.71 \pm-2.01$ & $-14.65 \pm-2.19$ & 0.013 \\
\hline & $A P 4 \%$ & $-15.83 \pm-2.67$ & $-21.87 \pm-1.43$ & $<0.0001$ & $-16.98 \pm-1.82$ & $-14.96 \pm-2.92$ & 0.038 \\
\hline & $A P 2 \%$ & $-15.94 \pm-2.63$ & $-20.93 \pm-1.37$ & $<0.0001$ & $-17.03 \pm-2.25$ & $-15.11 \pm-2.28$ & 0.046 \\
\hline & $G L \% S$ & $-15.87 \pm-2.25$ & $-21.26 \pm-1.23$ & $<0.0001$ & $-16.90 \pm-1.80$ & $-15.08 \pm-2.28$ & 0.025 \\
\hline \multicolumn{2}{|l|}{ ABI average } & $0.59 \pm 0.15$ & $0.98 \pm 0.08$ & $<0.0001$ & $0.69 \pm-0.13$ & $0.51 \pm 0.11$ & $<0.0002$ \\
\hline
\end{tabular}

LV_EDD left ventricular end-diastolic dimension, LV_ESD left ventricular end-systolic dimension, LV_EDV left ventricular end-diastolic volume, LV_ESV left ventricular end-systolic volume, EF\% left ventricular ejection fraction, AP3\% apical three view left ventricular strain, AP4\% apical four view left ventricular strain, AP2\% apical two view left ventricular strain, GLS \% global longitudinal strain of left ventricle and ABI ankle brachial index.

\begin{tabular}{|c|c|c|c|c|c|}
\hline \multicolumn{6}{|c|}{ Table 2 Characteristics of patients according to severity } \\
\hline & & Some & Moderate & Severe & \multirow{2}{*}{ P- Value } \\
\hline & & Mean \pm SD & Mean \pm SD & Mean \pm SD & \\
\hline Age & & $55.50 \pm 11.09$ & $62.47 \pm 6.60$ & $66.86 \pm 5.18$ & 0.05 \\
\hline \multirow{5}{*}{$\begin{array}{l}\text { Conventional } \\
\text { Echo. }\end{array}$} & $L V \_E D D$ & $4.45 \pm 0.13$ & $4.26 \pm 0.28$ & $4.48 \pm 0.11$ & 0.096 \\
\hline & $L V \_E S D$ & $3.11 \pm 0.07$ & $3.17 \pm 0.15$ & $3.31 \pm 0.14$ & 0.045 \\
\hline & $L V \_E D V$ & $87.93 \pm 7.80$ & $78.39 \pm 15.43$ & $90.29 \pm 6.95$ & 0.105 \\
\hline & $L V \_E S V$ & $30.00 \pm 2.16$ & $32.00 \pm 1.59$ & $36.57 \pm 4.76$ & 0.04 \\
\hline & $E F \%$ & $65.50 \pm 5.07$ & $58.58 \pm 4.41$ & $59.43 \pm 5.44$ & 0.043 \\
\hline \multirow{4}{*}{$\begin{array}{l}\text { 2D Speckle } \\
\text { Echo. }\end{array}$} & AP3\% & $-18.05 \pm-2.08$ & $-15.81 \pm-1.98$ & $-13.37 \pm-1.49$ & 0.002 \\
\hline & AP4\% & $-18.45 \pm-0.82$ & $-16.26 \pm-2.32$ & $-13.17 \pm-2.12$ & 0.001 \\
\hline & $A P 2 \%$ & $-18.93 \pm-1.34$ & $-15.92 \pm-2.31$ & $-14.31 \pm-2.77$ & 0.015 \\
\hline & $G L S \%$ & $-18.48 \pm-1.01$ & $-15.99 \pm-1.99$ & $-14.04 \pm-1.88$ & 0.003 \\
\hline ABI Average & & $0.82 \pm 0.03$ & $0.61 \pm 0.10$ & $0.41 \pm 0.04$ & $<0.0001$ \\
\hline
\end{tabular}


Detection of Subclinical Left Ventricular Systolic dysfunction by 2D Speckle Tracking...

While other conventional echocardiography parameters there was no statistically significance with neither ABI nor severity.

There was statistically significance between 2D-STE parameters including AP3\%, $(\mathrm{P}$-value $=0.004, \mathrm{r}=0.51), \mathrm{AP} 4 \%(\mathrm{P}$-value $=$ $0.001, \mathrm{r}=0.57)$, AP2\% $(\mathrm{p}$-value $=0.005, \mathrm{r}=$ $0.50)$ and GLS\% (P-value $=0.003, r=0.52)$ and $\mathrm{ABI}$ average with a moderately strong positive correlation between both parameters (fig.1).

In terms of severity of $\mathrm{ABI}$ decrease, this significance was more affected in both limbs affected subgroup (table 1) than one limb affected subgroup regarding 2D-STE parameters including AP3\%, AP4\%, AP2\% and GLS\% (P-value $=0.013,0.038,0.046$ and 0.025 respectively). Also, this significance was more affected in severe arterial disease than moderate arterial disease than some arterial disease subgroups regarding 2D-STE parameters including AP3\%, AP4\%, AP2\% and GLS\% (P-value $=0.002,0.001,0.015$ and 0.003 respectively) (table 2 ).

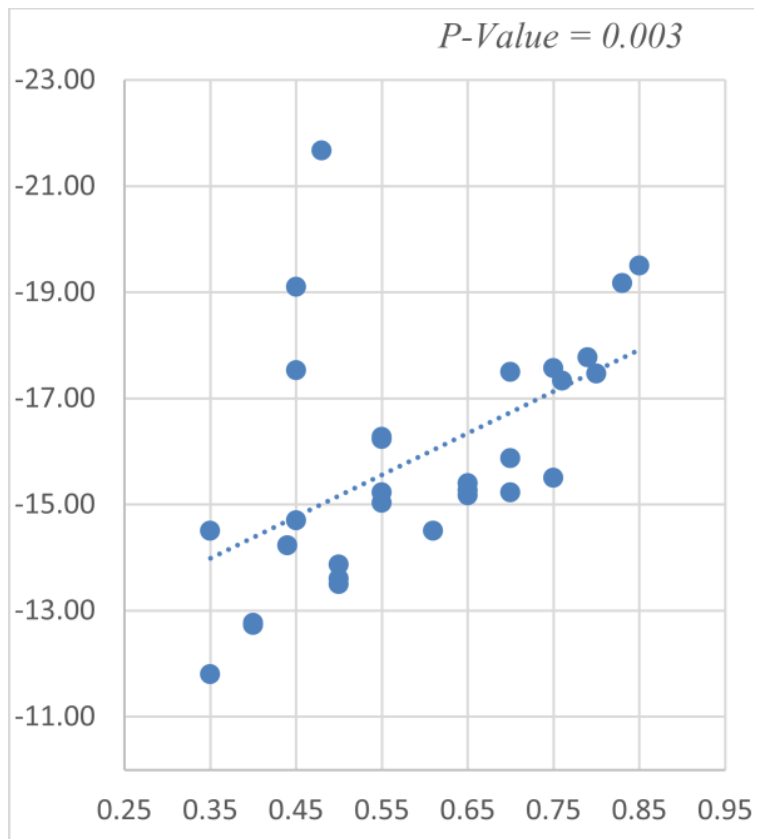

Fig.1. showing correlation between $A B I$ and GLS\%

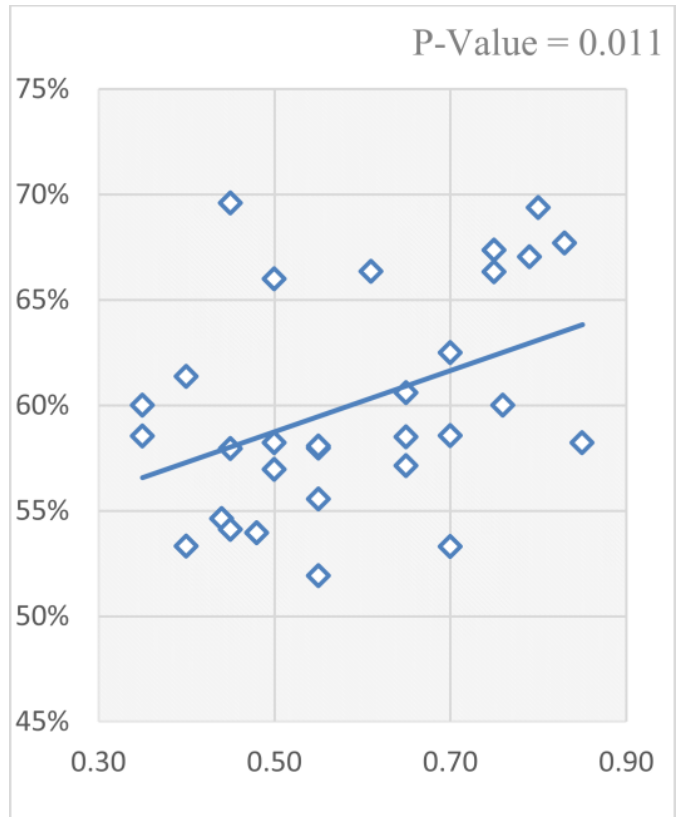

Fig.2. showing correlation between ABI and EF\%

\section{DISCUSSION}

Overall, LV dysfunction and heart failure are more frequent in patients with PAD. One-third of patients with symptomatic PAD have reduced left ventricular (LV) ejection fraction (12,23) and at least twice as prevalent in patients with PAD as in the general population, matched for age and sex. ${ }^{(\mathbf{2 4 , 2 5})}$ In a metaanalysis of 11,304 patients with PAD, Anand $\boldsymbol{e t} \boldsymbol{a l .},{ }^{(26)}$ found that the prevalence of heart failure in patients with PAD was $7.9 \%$, which was greater than the $4.1 \%$ expected from NHANES data. Thus, the prevalence of PAD is nearly doubled in the setting of heart failure, and PAD is associated with greater functional impairment and worse prognosis. Despite the high prevalence and incidence of heart failure in patients with PAD, outcome data for this group are very limited. Evaluation of LV function in PAD may be of value for better risk stratification for future $\mathrm{CV}$ events and comprehensive management of patients' CV diseases ${ }^{(25)}$. This is particularly important when an intermediate- or high-risk vascular intervention is planned ${ }^{(27)}$. 
Few previous studies have assessed the relation between LV systolic function and the ABI. Of 204 patients with symptomatic PAD, Ward et al., (12) found that decrease in LVEF was more common among patients with a low ABI than in those with a normal ABI. They found PAD to be an independent predictor of LV dysfunction. Thatipelli $\boldsymbol{e t}$ al. ${ }^{(28)}$ studied 395 patients referred for dobutamine stress echocardiography and ABI determination and found $\mathrm{ABI}$ to be the stronger predictor of all-cause mortality and it was unrelated to the LV wall motion index.

Similar to, Rizvi et al., ${ }^{(24)}$ had concluded that ABI was independently associated with LVEF in community patients referred for ABI studies. Compared with subjects with a normal $\mathrm{ABI}$, those with a low $\mathrm{ABI}$ had a lower LVEF and those with a high ABI had a higher LVEF. These findings were independent of the presence and severity of CAD. the relation between ABI and LVEF was independent of PAD symptom status. Also, it was found that in non-diabetic individuals those with a low ABI had a lower $\operatorname{LVEF}^{(29)}$.

To our knowledge no previous study studied detection of subclinical LV dysfunction in peripheral arterial disease and effect of its severity. The main finding of this study that the global and segmental longitudinal stain was lower in peripheral arterial disease patients than in healthy one with lower strain in more lower ankle brachial index which denote subtle LV systolic dysfunction in peripheral arterial disease patient irrespective to risk factors as age, gender, hypertension, smoking or diabetes mellitus. Although, as far as we know the mechanism of heart failure in PAD is uncertain but it could be multi factorial. Coexistent $\mathrm{CAD}^{(30)}$ or microangiopathy together with diabetes, smoking and other risk factors, inflammation all may be factors leading to the development of heart failure in PAD patients.

Also, elevated aortic stiffness increases left ventricular (LV) afterload and high pulse pressure impairs coronary blood flow, resulting in hypertension, LV hypertrophy, diastolic dysfunction and ultimately heart failure ${ }^{(31,32)}$. Importantly, skeletal muscle involvement and deconditioning in PAD may affect heart failure severity. On the other hand, functional limitation due to heart failure is likely to mask symptoms of PAD, causing underestimation of the number of patients with both conditions $^{(2)}$.

The study found that there is moderate positive correlation between the Ankle brachial index and LVEF $(\mathrm{P}$-value $=0.011, \mathrm{r}=$ 0.47). Similar to recent study by Nunez et al. $^{(33)}$ in 2018 who studied the relation of the ankle brachial index to the left ventricular EF in 55 patients without CAD (normal coronary angiography, he found that $\mathrm{ABI}$ correlated with LVEF $(r=0.40, p=0.002)$ and his median LVEF was lower in subjects with low ABI values compared to those with normal $A B I$ values (33 vs. $61 \%$; $p=0.001$ )

Although there have been many studies addressing ventricular-arterial coupling, this study is the first study addresses this point using speckle-tracking imaging with LV GLS as a marker of LV longitudinal function and $\mathrm{ABI}$ as arterial stiffness marker. This study showed the independent association between ABI as a predictor for arterial stiffness and Left Ventricular global longitudinal strain (LV GLS), suggesting a close interaction between arterial stiffness and LV long-axis function. This study showed that even there is correlation between decline ABI and left ventricular systolic function $\mathrm{EF} \%$ but LV GLS offer a better way to detect subclinical dysfunction in asymptomatic patient.

This is indirectly consistent with another study that have been conducted on 248 subjects without structural heart problems, that used brachial-ankle pulse wave velocity (baPWV) another marker for arterial stiffness, that showed that there is a significant positive association with LV GLS and baPWV. $(\mathrm{P}=0.001)$ and concluded that may be there is independent linear correlation between LV GLS measured by 2D speckletracking imaging and baPWV, supporting the concept that increased arterial stiffness impairs LV performance ${ }^{(34)}$.

\section{STUDY LIMITATIONS}

Several potential limitations of this study should be considered. First, it had a relatively small number of patients. Second, regional, circumferential, radial strains were not evaluated in this study. Lastly, no functional testing to assess the severity of myocardial ischemia was performed. 
Detection of Subclinical Left Ventricular Systolic dysfunction by 2D Speckle Tracking...

\section{CONCLUSION}

Peripheral arterial disease patients had lower global and segmental longitudinal strain than the healthy subjects and this was more prominent either with severity or limb affection. This study showed that even there is correlation between decline ABI and left ventricular systolic function EF\% but LV GLS offer a better economic way to detect subclinical dysfunction in asymptomatic patient. Further multicenter and prospective studies are required to confirm our findings.

\section{REFERENCES}

1. Malyar NM, Freisinger E, Meyborg

$M$ et al. (2016): Low Rates of Revascularization and High In-Hospital Mortality in Patients With Ischemic Lower Limb Amputation. Angiology, 67(9):860-869.

2. Aboyans V, Ricco J-B, Bartelink M-LEL et al. (2018): 2017 ESC Guidelines on the Diagnosis and Treatment of Peripheral Arterial Diseases, in collaboration with the European Society for Vascular Surgery (ESVS). Eur Heart J., 39(9):763-816.

3. Espinola-Klein C, Rupprecht HJ, Bickel C et al. (2008): Different calculations of ankle-brachial index and their impact on cardiovascular risk prediction. Circulation, 118(9):961-967.

4. Fowkes FGR, Rudan D, Rudan I et al. (2013): Comparison of global estimates of prevalence and risk factors for peripheral artery disease in 2000 and 2010: A systematic review and analysis. Lancet, 382(9901):1329-1340.

5. Sigvant B, Lundin F, Wahlberg $\mathbf{E}$ (2016): The Risk of Disease Progression in Peripheral Arterial Disease is Higher than Expected: A Meta-Analysis of Mortality and Disease Progression in Peripheral Arterial Disease. Eur J Vasc Endovasc Surg., 51(3):395-403.

6. Norgren L, Hiatt WR, Dormandy JA, Nehler MR, Harris KA, Fowkes F (2007): Inter-Society Consensus for the Management of Peripheral Arterial Disease (TASC II). J Vasc Surg., 45(1):S5-S67.

7. Hirsch AT, Haskal ZJ, Hertzer NR et al. (2006): ACC/AHA 2005 practice guidelines for the management of patients with peripheral arterial disease (lower extremity, renal, mesenteric, and abdominal aortic): Executive summary. Circulation, 113(11):1474-1547.

8. Majesky MW (2007): Developmental
Basis of Vascular Smooth Muscle Diversity. Arterioscler Thromb Vasc Biol., 27(6):1248-1258.

9. Chen HI, Sharma B, Akerberg BN et al. (2014): The sinus venosus contributes to coronary vasculature through VEGFCstimulated angiogenesis. Development, 141(23):4500-4512.

10. Red-Horse K, Ueno H, Weissman IL, Krasnow MA (2010): Coronary arteries form by developmental reprogramming of venous cells. Nature, 464(7288):549-553.

11. Sharma B, Ho L, Ford GH et al. (2017): Alternative Progenitor Cells Compensate to Rebuild the Coronary Vasculature in Elabela- and Apj-Deficient Hearts. Dev Cell, 42(6):655-666.e3.

12. Ward RP, Goonewardena SN, Lammertin G, Lang RM (2007): Comparison of the Frequency of Abnormal Cardiac Findings by Echocardiography in Patients With and Without Peripheral Arterial Disease. Am J Cardiol., 99(4):499503.

13. Gupta DK, Skali H, Claggett B et al. (2014): Heart failure risk across the spectrum of ankle-brachial index: The ARIC study (Atherosclerosis RiskIn Communities). JACC Hear Fail, 2(5):447454.

14. Norman PE, Eikelboom JW, Hankey GJ (2004): Peripheral arterial disease: prognostic significance and prevention of atherothrombotic complications. Med J Aust., 181(3): 150-154.

15. FU W, YE C, MEI C, RONG S, WANG W (2006): Reverse correlation between ankle-brachial index and left ventricular hypertrophy in patients on maintenance haemodialysis. Nephrology, 11(1):9-14.

16. Hunt SA, Abraham WT, Chin MH et al. (2005): ACC/AHA 2005 Guideline Update for the Diagnosis and Management of Chronic Heart Failure in the Adult. Circulation, 112(12):38-43.

17. Hoffmann S, Jensen JS, Iversen AZ et al. (2012): Tissue Doppler echocardiography improves the diagnosis of coronary artery stenosis in stable angina pectoris. Eur Hear J - Cardiovasc Imaging, 13(9):724-729.

18. Bell V, McCabe EL, Larson MG et al. (2017): Relations Between Aortic Stiffness and Left Ventricular Mechanical Function in the Community. J Am Heart Assoc., 6(1). 
19. Russo C, Jin Z, Elkind MSV et al. (2014): Prevalence and prognostic value of subclinical left ventricular systolic dysfunction by global longitudinal strain in a community-based cohort. Eur J Heart Fail, 16(12):1301-1309.

20. Cheng S, McCabe EL, Larson MG et al. (2015): Distinct Aspects of Left Ventricular Mechanical Function Are Differentially Associated With Cardiovascular Outcomes and All-Cause Mortality in the Community. J Am Heart Assoc., 4(10):e002071.

21. Kalam K, Otahal P, Marwick TH (2014): Prognostic implications of global LV dysfunction: a systematic review and meta-analysis of global longitudinal strain and ejection fraction. Heart, 100(21):16731680.

22. Lang RM, Badano LP, Mor-Avi V et al. (2015): Recommendations for Cardiac Chamber Quantification by Echocardiography in Adults: An Update from the American Society of Echocardiography and the European Association of Cardiovascular Imaging. Eur Hear $\mathbf{J}$ - Cardiovasc Imaging, 16(3):233-271.

23. Kelly R, Staines A, MacWalter $\mathbf{R}$, Stonebridge $\mathbf{P}$, Tunstall-Pedoe $\mathrm{H}$, Struthers AD (2002): The prevalence of treatable left ventricular systolic dysfunction in patients who present with noncardiac vascular episodes. J Am Coll Cardiol., 39(2):219-224.

24. Rizvi S, Kamran H, Salciccioli L, Saiful F, Lafferty J, Lazar JM (2010): Relation of the Ankle Brachial Index to Left Ventricular Ejection Fraction. Am J Cardiol., 105(1):129-132.

25. Hedberg P, Hammar C, Selmeryd J et al. (2014): Left ventricular systolic dysfunction in outpatients with peripheral atherosclerotic vascular disease: Prevalence and association with location of arterial disease. Eur J Heart Fail, 16(6):625-632.
26. Anand RG, Ventura HO, Mehra MR (2007): Is Heart Failure More Prevalent in Patients With Peripheral Arterial Disease? A Meta-Analysis. Congest Hear Fail, 13(6):319-322.

27. Kristensen SD, Knuuti J, Saraste A et al. (2014): 2014 ESC/ESA Guidelines on noncardiac surgery: cardiovascular assessment and management. Eur Heart J., 35(35):2383-2431.

28. Thatipelli MR, Pellikka PA, McBane RD et al. (2007): Prognostic value of anklebrachial index and dobutamine stress echocardiography for cardiovascular morbidity and all-cause mortality in patients with peripheral arterial disease. J Vasc Surg., 46(1):62-70.

29. Abbasnezhad M, Aliasgarzadeh A, Aslanabadi H, Habibzadeh A, Zamani B (2011): Relation of ankle brachial index to left ventricular ejection fraction in nondiabetic individuals. J Cardiovasc Thorac Res., 3(4): 109-112.

30. Ding Y-M, Wang Y, Li Y et al. (2011): Association of ankle-brachial index with clinical coronary heart disease, stroke in aged Chinese hypertensive men. Chinese J Appl Physiol., 27(2):129-133.

31. O'Rourke MF, Safar ME, Dzau V (2010): The Cardiovascular Continuum extended: Aging effects on the aorta and microvasculature. Vasc Med., 15(6):461468.

32. Kahan T. (2012): The importance of myocardial fibrosis in hypertensive heart disease. J Hypertens, 30(4):685-687.

33. Nunez A, Russ S, Ihsan M et al. (2018): Relation of the Ankle Brachial Index to Left Ventricular Ejection Fraction in Patients Without Coronary Artery Disease. Cardiology, 141(1):18-24.

34. Kim H, Seo J, Chung W et al. (2015): Independent association between brachialankle pulse wave velocity and global longitudinal strain of left ventricle. Int J Cardiovasc Imaging, 31(8):1563-1570. 